\title{
In-medium modified energy-momentum tensor form factors of the nucleon within the framework of a $\pi-\rho-\omega$ soliton model
}

\author{
Ju-Hyun Jung, Ulugbek Yakhshiev, and Hyun-Chul Kim \\ Department of Physics, Inha University, Incheon 402-751, Republic of Korea \\ Peter Schweitzer \\ Department of Physics, University of Connecticut, Storrs, CT 06269, U.S.A.
}

\begin{abstract}
We investigate the energy-momentum tensor form factors of the nucleon in nuclear medium, based on an in-medium modified $\pi-\rho-\omega$ soliton model, with medium modifications of the mesons considered. The results allow us to establish general features of medium modifications of the structure of nucleons bound in a nuclear medium.

PACS numbers: 12.39.Dc, 21.65.Cd, 21.65.Jk

Keywords: Energy-momentum tensor form factors, Solitonic model, Mesons in nuclear matter.
\end{abstract}

\section{INTRODUCTION}

The energy-momentum tensor (EMT) form factors (FFs) provide a new aspect on the structure of the nucleon, since they contain essential information on how the constituents of the nucleon behave inside a nucleon. The EMTFFs have drawn considerable attention only very recently, even though they were first proposed by Pagels several decades ago [1]. The reason lies in the fact that the natural probe to access the EMTFFs is the graviton, which is by no means a tractable tool to measure them experimentally. In the meantime the generalized parton distributions (GPDs) paved the way for novel understanding of the inner structure of the nucleon [2-5]. The EMTFFs are given by Mellin moments of certain GPDs and characterize how mass, spin and internal forces are distributed inside a nucleon [3, 6, 7]. The EMTFFs are as fundamental as for instance the electromagnetic form factors but provide different insights.

The EMTFFs of the nucleon parametrize the nucleon matrix elements of the symmetric EMT operator as follows [6, 7]:

$$
\left\langle p^{\prime}\left|\hat{T}_{\mu \nu}(0)\right| p\right\rangle=\bar{u}\left(p^{\prime}, s^{\prime}\right)\left[M_{2}(t) \frac{P_{\mu} P_{\nu}}{M_{N}}+J(t) \frac{i\left(P_{\mu} \sigma_{\nu \rho}+P_{\nu} \sigma_{\mu \rho}\right) \Delta^{\rho}}{2 M_{N}}+d_{1}(t) \frac{\Delta_{\mu} \Delta_{\nu}-g_{\mu \nu} \Delta^{2}}{5 M_{N}}\right] u(p, s)
$$

where $P=\left(p+p^{\prime}\right) / 2, \Delta=\left(p^{\prime}-p\right)$ and $t=\Delta^{2}$. The $M_{N}$ and $u(p, s)$ denote the mass and the spinor of the nucleon, respectively. The form factor $M_{2}(t)$ is related to the distribution of the energy density inside the nucleon. The quark and gluon contributions to this form factor at zero-momentum transfer are known from studies of deep-inelastic scattering and tell us that about a half of the momentum of a fast moving nucleon is carried by quarks, and the other half by gluons. The form factor $J(t)$ is related to the total angular momentum of quarks and gluons, and is not known experimentally. It is equally important to understand the form factor $d_{1}(t)$ in Eq. (1), since it describes how the strong forces are distributed and stabilized in the nucleon [7, 8]. In all theoretical studies so far the value of this form factor at zero-momentum transfer, $d_{1} \equiv d_{1}(0)$, was found to have a negative sign, and it was argued that this fact is deeply rooted in the spontaneous breakdown of chiral symmetry [11]. Information on EMTFFs can be obtained from studies of hard exclusive reactions, and in particular on $d_{1}(t)$ from the beam charge asymmetry in deeply virtual Compton scattering.

The EMTFFs of the free nucleon were studied in the bag model [12] and in soliton models in Refs. [13 17], and most recently in the framework of the $\pi-\rho-\omega$ solitonic model [18]. This model highlighted the role of $\rho$ and $\omega$-mesons in describing the nucleon structure. As known from the one-boson exchange potential for the nucleon-nucleon interaction, the vector mesons provide short-range repulsive forces, while the pion degrees of freedom furnish long-range attractive ones [19]. The EMTFF $d_{1}(t)$ accommodates the ideal ground to study the interplay of the attractive and repulsive forces, which must exactly balance each other to comply with stability requirements. The characteristics of the vector mesons in the $\pi-\rho-\omega$ solitonic model turned out to be very similar to the one-boson exchange potential: The pion provides attraction at large distances, which is exactly balanced by repulsion at short distances due to vector mesons.

The nucleon is known to undergo changes in nuclear medium due to its environment. The EMTFFs feature essential information on how the nucleon is modified in 
medium. Thus, it is very important to examine the medium modification of the EMTFFs of the nucleon. These form factors have been studied so far only in the framework of the medium-modified Skyrme-model [20]. In this context, it is of great importance to extend the recent investigation in the chiral solitonic model with explicit $\pi, \rho$ and $\omega$ mesonic degrees of freedom [18] to nuclear matter based on a medium-modified $\pi-\rho-\omega$ soliton model 21] and to study how the structure of the nucleon undergoes changes due to the surrounding nuclear environment. The EMTFFs will directly reveal how the changes of the $\pi$, the $\rho$, and the $\omega$ in medium will affect the properties of the nucleon. This is our main purpose in the present work. Moreover, the studies of nuclear medium effects may shed light on the EMTFFs of nuclei for which conflicting theoretical predictions exist [22 24]. The first measurements of deeply virtual Compton scattering on nuclei by HERMES [25] did not reach the level of accuracy required to resolve nuclear effects. But future experiments at Jefferson Lab may provide new insights into the way how the nucleon is modified in medium.

The present work is organized as follows: In Section II, we briefly explain the formalism of the medium-modified $\pi-\rho-\omega$ soliton model. In Section III, we derive the expressions for the EMTFFs within the present framework. In Section IV, we discuss the results of the EMTFFs. In the final Section we summarize and draw conclusions. Appendix contains an alternative general proof of the stability condition for the $\pi-\rho-\omega$ soliton model.

\section{GENERAL FORMALISM}

We start from the in-medium modified effective chiral Lagrangian with the $\pi, \rho$, and $\omega$ meson degrees of freedom, where the nucleon arises as a topological soliton. Using the asterisk to indicate medium modified quantities, the Lagrangian has the form

$$
\mathcal{L}^{*}=\mathcal{L}_{\pi}^{*}+\mathcal{L}_{V}^{*}+\mathcal{L}_{\mathrm{kin}}^{*}+\mathcal{L}_{\mathrm{WZ}}^{*},
$$

where the corresponding terms are expressed as

$$
\begin{aligned}
\mathcal{L}_{\pi}^{*}= & \frac{f_{\pi}^{2}}{4} \operatorname{Tr}\left(\partial_{0} U \partial_{0} U^{\dagger}\right)-\alpha_{p} \frac{f_{\pi}^{2}}{4} \operatorname{Tr}\left(\partial_{i} U \partial_{i} U^{\dagger}\right) \\
& +\alpha_{s} \frac{f_{\pi}^{2} m_{\pi}^{2}}{2} \operatorname{Tr}(U-1) \\
\mathcal{L}_{V}^{*}= & \frac{f_{\pi}^{2}}{2} \operatorname{Tr}\left[D_{\mu} \xi \cdot \xi^{\dagger}+D_{\mu} \xi^{\dagger} \cdot \xi\right]^{2} \\
\mathcal{L}_{\mathrm{kin}}^{*}= & -\frac{1}{2 g_{V}^{2} \zeta_{V}} \operatorname{Tr}\left(F_{\mu \nu}^{2}\right) \\
\mathcal{L}_{\mathrm{WZ}}^{*}= & \left(\frac{N_{c}}{2} g_{\omega} \sqrt{\zeta_{\omega}}\right) \omega_{\mu} \frac{\epsilon^{\mu \nu \alpha \beta}}{24 \pi^{2}} \\
& \times \operatorname{Tr}\left\{\left(U^{\dagger} \partial_{\nu} U\right)\left(U^{\dagger} \partial_{\alpha} U\right)\left(U^{\dagger} \partial_{\beta} U\right)\right\} .
\end{aligned}
$$

Here, the $\mathrm{SU}(2)$ chiral field is written as $U=\xi_{L}^{\dagger} \xi_{R}$ in unitary gauge, and the field-strength tensor and the co- variant derivative are defined, respectively, as

$$
\begin{aligned}
F_{\mu \nu} & =\partial_{\mu} V_{\nu}-\partial_{\nu} V_{\mu}-i\left[V_{\mu}, V_{\nu}\right], \\
D_{\mu} \xi_{L(R)} & =\partial_{\mu} \xi_{L(R)}-i V_{\mu} \xi_{L(R)},
\end{aligned}
$$

where the vector field $V_{\mu}$ includes the $\rho$-meson and $\omega$ meson fields, i.e. $\boldsymbol{\rho}_{\mu}$ and $\omega_{\mu}$, respectively, expressed as

$$
V_{\mu}=\frac{g_{V} \sqrt{\zeta_{V}}}{2}\left(\boldsymbol{\tau} \cdot \boldsymbol{\rho}_{\mu}+\omega_{\mu}\right)
$$

with the Pauli matrices $\boldsymbol{\tau}$ in isospin space.

Note that in Eqs. (41), (5) and (9) subscript $V$ generically stands for both the $\rho$-meson and the $\omega$-meson and for compactness we keep the generic form of those expressions. One can separate Eqs. (4) and (5) into the $\rho$ and $\omega$-meson parts using the definitions (77), (8) and (9). Then $g_{V}$ appearing in the $\rho$-meson part denotes $g_{\rho}$, and $g_{V}$ in the $\omega$-meson part designates $g_{\omega}$. Finally, $N_{c}=3$ is the number of colors.

Now let us discuss the parameters of the model appearing in the Lagrangian in Eqs. (3)-(6). They can be classified into two different classes: (i) some of the parameters $f_{\pi}, m_{\pi}, g_{\rho}, g_{\omega}$ and $N_{c}$ are related to the quantities in free space while (ii) the other parameters $\alpha_{p}, \alpha_{s}$ and $\zeta_{V}$ are pertinent to nuclear matter properties. ${ }^{1}$

In free space $\alpha_{p}=\alpha_{s}=\zeta_{\omega}=\zeta_{\rho}=1$ and the free-space parameters are fixed by using either experimental or empirical data on pions and vector mesons [26]. The pion decay constant and mass are taken to be $f_{\pi}=93 \mathrm{MeV}$ and $m_{\pi}=135 \mathrm{MeV}$ (the neutral pion mass). The values of the coupling constants for the $\rho$ and $\omega$ mesons are given respectively as $g_{\rho}=5.86$ and $g_{\omega}=5.95$. The Kawarabayashi-Suzuki-Riazuddin-Fayyazuddin (KSRF) relation connects them to the vector meson masses, i.e. $m_{\rho}=770 \mathrm{MeV}$ and $m_{\omega}=782 \mathrm{MeV}$, as follows

$$
2 f_{\pi}^{2} g_{\rho}^{2}=m_{\rho}^{2}, \quad 2 f_{\pi}^{2} g_{\omega}^{2}=m_{\omega}^{2} .
$$

In general, the parameters $\alpha_{p}, \alpha_{s}$, and $\zeta_{V}$ stand for the medium functionals which are the essential quantities in the present work. They depend on the nuclear matter density $\rho$ and are defined as

$$
\begin{aligned}
& \alpha_{p}(\rho)=1-\frac{4 \pi c_{0} \rho / \eta}{\left.1+g_{0}^{\prime} 4 \pi c_{0} \rho\right) / \eta}, \\
& \alpha_{s}(\rho)=1-4 \pi \eta b_{0} \rho m_{\pi}^{-2}, \\
& \zeta_{V}(\rho)=\exp \left\{-\frac{\gamma_{\text {num }} \rho}{1+\gamma_{\operatorname{den}} \rho}\right\} .
\end{aligned}
$$

They provide crucial information on how the nuclearmatter environment influences properties of the single soliton [21]. The $\eta$ is a kinematic factor defined as $\eta=1+m_{\pi} / m_{N} \simeq 1.14$. The values of the empirical parameters $b_{0}=-0.024 m_{\pi}^{-1}$ and $c_{0}=0.09 m_{\pi}^{-3}$ are taken

\footnotetext{
${ }^{1} \zeta_{V}$ denotes also a generic form for both $\zeta_{\rho}$ and $\zeta_{\omega}$ which appear in the corresponding $\rho$ - and $\omega$-meson parts of the Lagrangian.
} 
from the analysis of pionic atoms and the data on lowenergy pion-nucleus scattering. The $g_{0}^{\prime}=0.7$ denotes the Lorentz-Lorenz factor that takes into account the shortrange correlations [27].

The additional parameters $\gamma_{\text {num }}$ and $\gamma_{\text {den }}$ are introduced phenomenologically to reproduce the saturation point at normal nuclear matter. Two different techniques have been discussed in literature [21], in order to introduce nuclear modifications in the present soliton approach, and in this work we will explore both models.

Model I: Here one neglects the small mass difference of the $\rho$ - and $\omega$-mesons in free space $\left(m_{\omega}=m_{\rho}=770 \mathrm{MeV}\right.$, $\left.g_{\omega}=g_{\rho}=5.86\right)$ and assumes that the KSRF relation still holds in nuclear matter

$$
2 f_{\pi}^{2} g_{\rho}^{2} \zeta_{\rho}=m_{\rho}^{* 2}=m_{\omega}^{* 2}, \quad \zeta_{\rho}=\zeta_{\omega} \neq 1 .
$$

Model II: Here we remove the degeneracy of the vector meson masses in free space $\left(m_{\rho} \neq m_{\omega}=782 \mathrm{MeV}\right.$, $\left.g_{\rho} \neq g_{\omega}=5.95\right)$, and instead of Eq. (12) assume that the KSRF relation is valid only for the $\rho$ meson, with the $\omega$ meson kept as in free space:

$$
2 f_{\pi}^{2} g_{\rho}^{2} \zeta_{\rho}=m_{\rho}^{* 2} \neq m_{\omega}^{* 2}, \quad \zeta_{\rho} \neq 1, \quad \zeta_{\omega}=1 .
$$

The two different techniques to implement nuclear modifications within the current approach reflect the possibility that the $\rho$ - and $\omega$-degrees of freedom could respond differently to a nuclear medium environment 28 , 29]. The effects of the $\omega$-mesons are mainly limited to the inner core of the nucleon. Therefore, the two variants describe the situation that the inner core of the nucleon is more (Model I) or less (Model II) affected by medium effects. The latter is a plausible scenario, at least around the normal nuclear matter density.

In practice, these two models yield comparable results in many respects. A notable (and in our context important) difference though, is the description of the incom- pressibility of symmetric nuclear matter: Model I yields a smaller value of the incompressibility, while Model II produces a larger one. It means that Model II gives a stiffer nuclear binding energy and agrees better with the data [21]. In both models the values of $\gamma_{\text {num }}$ and $\gamma_{\text {den }}$ are fitted to reproduce the coefficient of the volume term in the empirical mass formula $a_{V} \approx 26 \mathrm{MeV}$. Although this is larger than the experimental value $a_{V}^{\exp } \approx 16 \mathrm{MeV}$, the relative change of the in-medium nucleon mass is reproduced correctly. (See Eq. (12) in Ref. 21] and the corresponding explanation.) In Model I we have $\gamma_{\text {num }}=$ $2.390 m_{\pi}^{-3}$ and $\gamma_{\text {den }}=1.172 m_{\pi}^{-3}$, whereas in Model II we employ $\gamma_{\text {num }}=1.970 m_{\pi}^{-3}$ and $\gamma_{\text {den }}=0.841 m_{\pi}^{-3}$. For further details on these two models in relation to nuclear matter properties, we refer to Ref. [21].

Since we are interested in homogeneous and symmetric nuclear matter, the nuclear matter density can be regarded as a constant and the spherically symmetric hedgehog Ansätze can be employed. (In this situation the medium functionals $\alpha_{p}, \alpha_{s}, \zeta_{V}$ become ordinary functions of the nuclear matter density $\rho$.) With the notation $\hat{\boldsymbol{n}}=\boldsymbol{x} / r$ and $r=|\boldsymbol{x}|$, the chiral soliton and the vector fields can be expressed in terms of the radial profile functions $F(r), G(r)$, and $\omega(r)$ :

$$
\begin{aligned}
U & =\exp \{i \boldsymbol{\tau} \cdot \hat{\boldsymbol{n}} F(r)\}, \\
\rho_{\mu}^{a} & =\frac{\varepsilon_{0 \mu k a} \hat{n}_{k}}{g_{\rho} \sqrt{\zeta_{\rho}} r} G(r), \\
\omega_{\mu} & =\omega(r) \delta_{\mu 0} .
\end{aligned}
$$

Note that the presence of the factor $\left(g_{\rho} \sqrt{\zeta_{\rho}}\right)^{-1}$ in $\rho_{i}^{a}$ is essential to keep the same boundary conditions for the medium-modified profile function $G(r)$ as in free space, i.e. $G(0)=-2$.

Utilizing the hedgehog Ansätze, we are able to derive the static energy functional from the Lagrangian, which is identified as the classical soliton mass:

$$
\begin{aligned}
M_{\mathrm{sol}}^{*}= & 4 \pi \int_{0}^{\infty} \mathrm{d} r r^{2}\left\{\alpha_{p} f_{\pi}^{2}\left(\frac{\sin ^{2} F}{r^{2}}+\frac{F^{\prime 2}}{2}\right)+\alpha_{s} f_{\pi}^{2} m_{\pi}^{2}(1-\cos F)+\frac{2 f_{\pi}^{2}}{r^{2}}(1-\cos F+G)^{2}-\zeta_{\omega} g_{\omega}^{2} f_{\pi}^{2} \omega^{2}\right. \\
& \left.+\frac{1}{g_{\rho}^{2} \zeta_{\rho} r^{2}}\left(G^{2}+\frac{G^{2}(G+2)^{2}}{2 r^{2}}\right)-\frac{1}{2} \omega^{\prime 2}+\left(\frac{3}{2} g_{\omega} \sqrt{\zeta_{\omega}}\right) \frac{1}{2 \pi^{2} r^{2}} \omega \sin ^{2} F F^{\prime}\right\},
\end{aligned}
$$

where $f^{\prime}=\partial f / \partial r$, generically.

The next step is to minimize the classical soliton mass. This is done by solving the equations of motion for each meson field, which are derived as

$F^{\prime \prime}=-\frac{2}{r} F^{\prime}+\frac{1}{\alpha_{p} r^{2}}\left(4(G+1) \sin F-\left(2-\alpha_{p}\right) \sin 2 F\right)$

$$
\begin{gathered}
+\frac{\alpha_{s} m_{\pi}^{2}}{\alpha_{p}} \sin F-\frac{3 g_{\omega} \sqrt{\zeta_{\omega}}}{4 \pi^{2} \alpha_{p} f_{\pi}^{2}} \frac{\sin ^{2} F \omega^{\prime}}{r^{2}} \\
G^{\prime \prime}=2 f_{\pi}^{2} g_{\rho}^{2} \zeta_{\rho}(G+1-\cos F)+\frac{(G+2)(G+1) G}{r^{2}}, \\
\omega^{\prime \prime}=-\frac{2}{r} \omega^{\prime}+2 f_{\pi}^{2} g_{\omega}^{2} \zeta_{\omega} \omega-\frac{3 g_{\omega} \sqrt{\zeta_{\omega}}}{4 \pi^{2} r^{2}} F^{\prime} \sin ^{2} F
\end{gathered}
$$

with the corresponding boundary conditions

$$
F(0)=\pi, \quad G(0)=-2, \quad \omega^{\prime}(0)=0,
$$




$$
F(\infty)=G(\infty)=\omega(\infty)=0 .
$$

Having quantized the soliton collectively, we obtain

$$
\begin{aligned}
U(\boldsymbol{r}, t) & =A(t) U(\boldsymbol{r}) A^{+}(t), \\
\omega_{i}(\vec{r}, t)= & \frac{\Phi(r)}{r}\left(\boldsymbol{K} \times \frac{\boldsymbol{r}}{r}\right)_{i}, \\
\boldsymbol{\tau} \cdot \boldsymbol{\rho}_{\mathbf{0}}(\boldsymbol{r}, t)= & \frac{2}{g_{\rho}} A(t) \boldsymbol{\tau} \cdot\left[\boldsymbol{K} \xi_{1}(r)\right. \\
& \left.\quad+\hat{\boldsymbol{n}}(\boldsymbol{K} \cdot \hat{\boldsymbol{n}}) \xi_{2}(r)\right] A^{+}(t),
\end{aligned}
$$

$$
\boldsymbol{\tau} \cdot \boldsymbol{\rho}_{i}(\boldsymbol{r}, t)=A(t) \boldsymbol{\tau} \cdot \boldsymbol{\rho}_{i}(\boldsymbol{r}) A^{+}(t),
$$

where $2 \boldsymbol{K}$ denotes the angular velocity of the soliton with the relation $i \boldsymbol{\tau} \cdot \boldsymbol{K}=A^{+} \dot{A}$. This leads to the timedependent collective Hamiltonian

$$
H(t)=M_{\text {sol }}^{*}+\lambda^{*} \operatorname{Tr}\left(\dot{A} \dot{A}^{+}\right),
$$

where $\lambda^{*}$ denotes the moment of inertia for the rotating soliton

$$
\begin{aligned}
\lambda^{*}=4 \pi \int_{0}^{\infty} d r & \left\{\frac{2}{3} f_{\pi}^{2} r^{2}\left(\sin ^{2} F+8 \sin ^{4} \frac{F}{2}-8 \xi_{1} \sin ^{2} \frac{F}{2}+3 \xi_{1}^{2}+2 \xi_{1} \xi_{2}+\xi_{2}^{2}\right)\right. \\
+\frac{1}{3 g_{\rho}^{2} \zeta_{\rho}} & \left(4 G^{2}\left(\xi_{1}^{2}+\xi_{1} \xi_{2}-2 \xi_{1}-\xi_{2}+1\right)+2\left(G^{2}+2 G+2\right) \xi_{2}^{2}+r^{2}\left(3 \xi_{1}^{\prime 2}+\xi_{2}^{\prime 2}+2 \xi_{1}^{\prime} \xi_{2}^{\prime}\right)\right) \\
& \left.-\frac{1}{6}\left(\Phi^{\prime 2}+\frac{2 \Phi^{2}}{r^{2}}+2 f_{\pi}^{2} g_{\omega}^{2} \zeta_{\omega} \Phi^{2}\right)+g_{\omega} \sqrt{\zeta_{\omega}} \frac{\Phi F^{\prime}}{2 \pi^{2}} \sin ^{2} F\right\} .
\end{aligned}
$$

In the large $N_{c}$ limit, we are able to minimize the moment of inertia, so that we derive the coupled nonlinear differential equations for the next-order profile functions $\xi_{1}, \xi_{2}$ and $\Phi$

$$
\begin{aligned}
& \xi_{1}^{\prime \prime}=2 f_{\pi}^{2} g_{\rho}^{2} \zeta_{\rho}\left(\cos F+\xi_{1}-1\right) \\
&+\frac{G^{2}\left(\xi_{1}-1\right)+2(G+1) \xi_{2}}{r^{2}}-\frac{2 \xi_{1}^{\prime}}{r} \\
& \xi_{2}^{\prime \prime}=2 f_{\pi}^{2} g_{\rho}^{2} \zeta_{\rho}\left(-\cos F+\xi_{2}+1\right)-\frac{2 \xi_{2}^{\prime}}{r} \\
&+\frac{G^{2}\left(\xi_{1}-1\right)+2[(G+3) G+3] \xi_{2}}{r^{2}} \\
& \Phi^{\prime \prime}=2 f_{\pi}^{2} g_{\omega}^{2} \zeta_{\omega} \Phi-\frac{3 g_{\omega} \sqrt{\zeta_{\omega}} F^{\prime} \sin ^{2} F}{2 \pi^{2}}+\frac{2 \Phi}{r^{2}}
\end{aligned}
$$

with the boundary conditions defined as

$$
\begin{aligned}
& \xi_{1}^{\prime}(0)=\xi_{2}^{\prime}(0)=\Phi(0)=0, \\
& \xi_{1}(\infty)=\xi_{2}(\infty)=\Phi(\infty)=0 .
\end{aligned}
$$

The boundary conditions for $\xi_{1}$ and $\xi_{2}$ are constrained to satisfy $2 \xi_{1}(0)+\xi_{2}(0)=2$ that remains unchanged in nuclear matter. The other details can be found in Ref. [21].

\section{ENERGY-MOMENTUM TENSOR FORM FACTORS}

Using the definition of the canonical EMT operator

$$
T^{\mu \nu *}=\frac{\partial \mathcal{L}^{*}}{\partial\left(\partial \phi_{a}\right)} \partial^{\nu} \phi_{a}-g^{\mu \nu} \mathcal{L}^{*}
$$

where $\phi_{a}$ are the relevant meson degrees of freedom and $g^{\mu \nu}=\operatorname{diag}(1,-1,-1,-1)$ is the metric tensor in Minkowski space, and the Lagrangian as defined in Eq. (2), we can derive the expressions for the densities of the EMT. The resulting EMT is symmetric and the expressions for its components are given as

$$
\begin{aligned}
T^{00 *}(r)= & \alpha_{p} \frac{f_{\pi}^{2}}{2}\left(2 \frac{\sin ^{2} F}{r^{2}}+F^{2}\right)+\alpha_{s} f_{\pi}^{2} m_{\pi}^{2}(1-\cos F)+\frac{2 f_{\pi}^{2}}{r^{2}}(1-\cos F+G)^{2}-f_{\pi}^{2} g_{\omega}^{2} \zeta_{\omega} \omega^{2} \\
& +\frac{1}{g_{\rho}^{2} \zeta_{\rho} r^{2}}\left(G^{\prime 2}+\frac{G^{2}(G+2)^{2}}{2 r^{2}}\right)-\frac{1}{2} \omega^{\prime 2}+\left(\frac{3}{2} g_{\omega} \sqrt{\zeta_{\omega}}\right) \frac{1}{2 \pi^{2} r^{2}} \omega \sin ^{2} F F^{\prime}
\end{aligned}
$$

$$
\begin{aligned}
& T^{0 i *}(\boldsymbol{r}, \boldsymbol{s})=\frac{e^{i l m} r^{l} s^{m}}{(\boldsymbol{s} \times \boldsymbol{r})^{2}} \rho_{J}^{*}(r), \\
& T^{i j *}(r)=s^{*}(r)\left(\frac{r^{i} r^{j}}{r^{2}}-\frac{1}{3} \delta^{i j}\right)+p^{*}(r) \delta^{i j},
\end{aligned}
$$

where $T_{00}(r)$ is called the energy density, which provides information on how the mass is distributed inside a nucleon. The vector $s$ represents the direction of the quanti- 
zation axis for the spin and coincides with the space part of the polarization vector of the nucleon in the rest frame. The densities $\rho_{J}^{*}(r), p^{*}(r)$ and $s^{*}(r)$ denote respectively the angular momentum, pressure and shear-force densities in nuclear matter, which are derived as

$$
\begin{aligned}
\rho_{J}^{*}(r)= & \frac{f_{\pi}^{2}}{3 \lambda^{*}}\left(\sin ^{2} F+8 \sin ^{4} \frac{F}{2}-4 \sin ^{2} \frac{F}{2} \xi_{1}\right)+\frac{1}{3 g_{\rho}^{2} \zeta_{\rho} r^{2} \lambda^{*}}\left[\left(2-2 \xi_{1}-\xi_{2}\right) G^{2}\right]+\frac{g_{\omega} \sqrt{\zeta_{\omega}}}{8 \pi^{2} \lambda^{*}} \Phi \sin ^{2} F F^{\prime} \\
p^{*}(r)= & -\frac{1}{6} \alpha_{p} f_{\pi}^{2}\left(F^{\prime 2}+2 \frac{\sin ^{2} F}{r^{2}}\right)-\alpha_{s} f_{\pi}^{2} m_{\pi}^{2}(1-\cos F)-\frac{2}{3 r^{2}} f_{\pi}^{2}(1-\cos F+G)^{2}+f_{\pi}^{2} g_{\omega}^{2} \zeta_{\omega} \omega^{2}+\frac{1}{6} \omega^{\prime 2} \\
& +\frac{1}{3 g_{\rho}^{2} \zeta_{\rho} r^{2}}\left(G^{\prime 2}+\frac{G^{2}(G+2)^{2}}{2 r^{2}}\right), \\
s^{*}(r)= & \alpha_{p} f_{\pi}^{2}\left(F^{\prime 2}-\frac{\sin ^{2} F}{r^{2}}\right)-\frac{2 f_{\pi}^{2}}{r^{2}}(1-\cos F+G)^{2}+\frac{1}{g_{\rho}^{2} \zeta_{\rho} r^{2}}\left(G^{\prime 2}-\frac{G^{2}(G+2)^{2}}{r^{2}}\right)-\omega^{\prime 2}
\end{aligned}
$$

We will follow Refs. [17, 20] to compute the EMTFFs in a consistent way, and consider for each quantity only the respective leading contribution in the large $N_{c}$ limit. Thus, the EMTFFs are derived as

$$
\begin{aligned}
M_{2}^{*}(t)-\frac{t d_{1}^{*}(t)}{5 M_{N}^{* 2}} & =\frac{1}{M_{N}^{*}} \int \mathrm{d}^{3} \boldsymbol{r} T_{00}^{*}(r) j_{0}(r \sqrt{-t}) \\
d_{1}^{*}(t) & =\frac{15 M_{N}^{*}}{2} \int \mathrm{d}^{3} \boldsymbol{r} p^{*}(r) \frac{j_{0}(r \sqrt{-t})}{t} \\
J^{*}(t) & =3 \int \mathrm{d}^{3} \boldsymbol{r} \rho_{J}^{*}(r) \frac{j_{1}(r \sqrt{-t})}{r \sqrt{-t}}
\end{aligned}
$$

where $j_{0}(z)$ and $j_{1}(z)$ represent the spherical Bessel functions of order 0 and 1 , respectively.

Some of the EMT densities are required to satisfy the following constraints

$$
\begin{aligned}
\frac{1}{M_{N}^{*}} \int \mathrm{d}^{3} \boldsymbol{r} T_{00}^{*}(r) & =M_{2}^{*}(0)=1, \\
\int \mathrm{d}^{3} \boldsymbol{r} \rho_{J}^{*}(r) & =J^{*}(0)=\frac{1}{2} \\
\int \mathrm{d}^{3} \boldsymbol{r} p^{*}(r) & =0 .
\end{aligned}
$$

Equation (37) means that the volume integral of the energy density reproduces the nucleon mass. The second constraint given in Eq. (38) indicates that the nucleon has spin $\frac{1}{2}$, and the third one (39) is the necessary condition for stability [31] of the nucleon and is also known as the von Laue condition 32 .

The nuclear medium modifies the dynamics inside the nucleon as compared to the free space case, which may result in quantitatively distinct features. However, the general soliton structure in medium is kept to be the same as in free space. The proofs of the relations (37[39) for a nucleon in medium, can therefore be step by step carried over from corresponding proofs given for a free nucleon in Ref. [18], and we do not repeat them here.
In Appendix $\mathrm{A}$ we give an alternative proof of the von Laue condition (39).

\section{RESULTS AND DISCUSSIONS}

In this Section, we present and discuss the numerical results. In Table I we list the results of the quantities relevant to the EMT densities and FFs in free space. One can see that both the results from Model I and Model II are very similar to each other, which indicates that the differences of the masses and the coupling constants between the $\rho$ meson and the $\omega$ meson parameters are not crucial in free space. The results from Model I were reported in Ref. [18].

The relative in-medium changes in the EMT densities and the EMTFFs are presented in Table [I] For comparison we include the results from the Skyrme model without (in Table I) and with (in Table II) medium modifications [20]. Depending on the quantity, the absolute numbers from the Skyrme model of Ref. [20] and the $\pi-\rho-\omega$ soliton model studied here differ significantly, see Table I. For instance, the energy density and the pressure in the center of the nucleon are lower in the Skyrme model [20] than those of the present work. In large part, these differences can be attributed to the smaller soliton mass $M_{\text {sol }}=881 \mathrm{MeV}$ in the Skyrme model [20] as compared to $1473 \mathrm{MeV}$ in the present work. However, the aim of this work is not to compare the Skyrme and $\pi-\rho$ $\omega$ soliton models per se. ${ }^{2}$ Rather this work is focused on studying nuclear medium effects. It is therefore a re-

\footnotetext{
2 That would require tuning model parameters to have more comparable soliton descriptions. For more discussion of Skyrme and $\pi-\rho-\omega$ soliton models for a free nucleon, from the point of view of EMT properties, see Ref. [18].
} 


\begin{tabular}{ccccccc}
\hline \hline & $\begin{array}{c}T_{00}(0) \\
{\left[\mathrm{GeV} / \mathrm{fm}^{3}\right]}\end{array}$ & $\begin{array}{c}\left\langle r_{00}^{2}\right\rangle \\
{\left[\mathrm{fm}^{2}\right]}\end{array}$ & $\begin{array}{c}\left\langle r_{J}^{2}\right\rangle \\
{\left[\mathrm{fm}^{2}\right]}\end{array}$ & $\begin{array}{c}p(0) \\
{\left[\mathrm{GeV} / \mathrm{fm}^{3}\right]}\end{array}$ & $\begin{array}{c}r_{0} \\
{[\mathrm{fm}]}\end{array}$ & $\begin{array}{c}d_{1}(0) \\
{\left[\mathrm{fm}_{F}^{2}\right\rangle}\end{array}$ \\
\hline Present work (Model I) & 3.56 & 0.78 & 0.74 & 0.58 & 0.55 & -5.03 \\
Present work (Model II) & 3.51 & 0.79 & 0.74 & 0.59 & 0.55 & -5.13 \\
Skyrme model [20] & 1.45 & 0.68 & 1.09 & 0.26 & 0.71 & -3.54 \\
\hline \hline
\end{tabular}

TABLE I: The quantities relevant to the nucleon EMT densities and their form factors for the free space nucleons: $T_{00}(0)$ is the energy density in the center of the nucleon; $\left\langle r_{00}^{2}\right\rangle$ is the mean-squared radius of the energy density; $\left\langle r_{J}^{2}\right\rangle$ represent that of the angular momentum distributions; $p(0)$ denotes the pressure value at the origin; $r_{0}$ designates the node of the pressure distribution such that $p\left(r_{0}\right)=0 ; d_{1}(0)$ corresponds to the $d_{1}(t)$ form factors at zero momentum transfer; and $\left\langle r_{F}^{2}\right\rangle$ is defined below in Eq. (43).

\begin{tabular}{cccccccc}
\hline \hline & $T_{00}^{*}(0) / T_{00}(0)$ & $\left\langle r_{00}^{2}\right\rangle^{*} /\left\langle r_{00}^{2}\right\rangle$ & $\left\langle r_{J}^{2}\right\rangle^{*} /\left\langle r_{J}^{2}\right\rangle$ & $p^{*}(0) / p(0)$ & $r_{0}^{*} / r_{0}$ & $d_{1}^{*}(0) / d_{1}(0)$ & $\left\langle r_{F}^{2}\right\rangle^{*} /\left\langle r_{F}^{2}\right\rangle$ \\
\hline Present work (Model I) & 0.61 & 1.36 & 1.04 & 0.59 & 1.18 & 1.11 \\
Present work (Model II) & 0.52 & 1.46 & 1.09 & 0.59 & 1.22 & 1.33 \\
Skyrme model [20] & 0.49 & 1.40 & 1.24 & 0.50 & 1.27 & 1.37 \\
\hline \hline
\end{tabular}

TABLE II: The ratio of the quantities at normal nuclear matter density $\rho=\rho_{0}$ to those in free space presented in Table 1 For comparison the results from the in-medium modified Skyrme model [20] are also presented.

markable observation that, in spite of numerically very different absolute descriptions, in Table凹, the relative impact of a nuclear environment on the properties of a single nucleon is qualitatively similar in the $\pi-\rho-\omega$ soliton- and Skyrme-model, see Table II

The energy density in the center of the nucleon, $T_{00}^{*}(0)$, is rather sensitive to the change of the nuclear matter density. The value of $T_{00}^{*}$ from Model $\mathrm{I}$ is reduced by around $40 \%$ and that from Model II decreases by almost $50 \%$. This is in line with the observations made in the Skyrme model [20], where $T_{00}^{*}$ also decreased by about $50 \%$. It indicates to some degree the well known fact that the nucleon mass tends to decrease in nuclear matter.

The energy mean-squared radius $\left\langle r_{00}^{2}\right\rangle^{*}$ is defined as

$$
\left\langle r_{00}^{2}\right\rangle^{*}=\frac{\int d^{3} \boldsymbol{r} r^{2} T_{00}^{*}(r)}{\int d^{3} \boldsymbol{r} T_{00}^{*}(r)} .
$$

As shown in Table [ the value of $\left\langle r_{00}^{2}\right\rangle^{*}$ increases from $0.78(0.79) \mathrm{fm}^{2}$ to $1.06(1.15) \mathrm{fm}^{2}$ in the case of Model I (Model II) when the density changes from zero to the normal nuclear matter density. The rate of the change in $\left\langle r_{00}^{2}\right\rangle^{*}$ is almost the same as in the case of the Skyrme model (see Table II). It implies that the size of the nucleon in medium generally increases. We will discuss the physical implications of other quantities along with the EMTFFs.

In the left panel of Fig. 1 we depict the results of the energy density normalized by the nucleon mass as functions of the radial distance $r$. While there is almost no difference between Model I and Model II in free space, we find that $T_{00}^{*}(r)$ in nuclear matter undergoes a larger change in Model II than in Model I. This can be traced back to the fact that the $\omega$ meson in Model II is assumed to remain intact in nuclear matter, while in Model I both the $\rho$ and $\omega$ meson masses undergo medium modifications. In general, the energy density is broadened and its maximum is reduced in nuclear medium. The broadening of the energy density can be more clearly seen in the density weighted by the factor $4 \pi r^{2}$ as shown in the right panel of Fig. 1. which results in the above-discussed increase of the energy mean-squared radius $\left\langle r_{00}^{2}\right\rangle^{*}$.

Figure 2 draws the spin densities of the nucleon as a function of $r$, normalized by its spin. As in the case of the energy densities, the spin density of the nucleon is also broadened in nuclear matter and its maximum is lessened. Note that the integration of the spin density over the space gives the spin of the nucleon, as defined in Eq. (38). This means that the decrease of the magnitude of the spin density in nuclear matter is compensated by the broadening of the density in such a way that the nucleon spin turns out be always one half. The spin mean-squared radius increases slightly in nuclear matter as shown in Table III, which is interesting because the $\left\langle r_{00}^{2}\right\rangle^{*}$ exhibits the increment in nuclear matter by about $40 \%$ whereas the $\left\langle r_{J}^{2}\right\rangle^{*}$ increases by less than $10 \%$.

Technically, the small changes in the angular momentum distribution can be understood from Eq. (31). One can see that the distribution corresponding to the external part of the soliton has no medium factor. This is due to the fact that the time-dependent part of the Lagrangian $\mathcal{L}_{\pi}^{*}$ is not modified in nuclear matter. As a result, the contribution from the outer shell of the rotating soliton remains more or less the same as in free space. Although the inner part of the soliton has explicit medium factors, clearly they will not lead to larger 

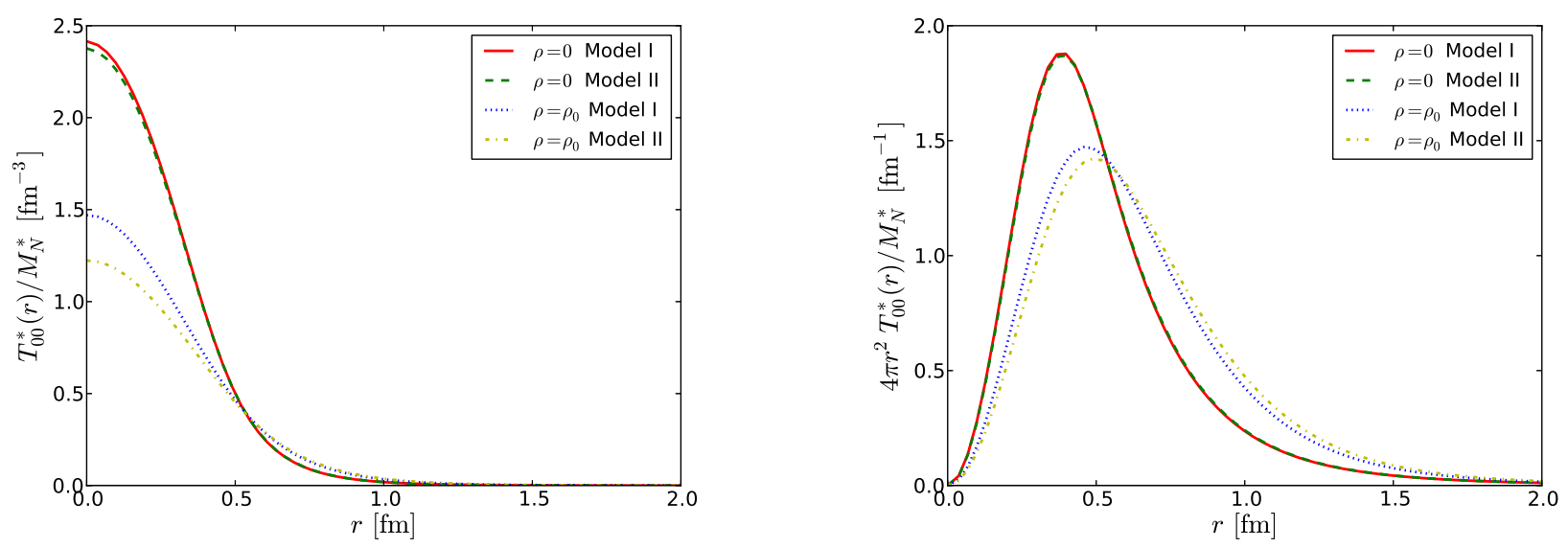

FIG. 1: The energy density in the nucleon normalized by the nucleon mass, $T_{00}^{*}(r) / M_{N}^{*}$ in the left panel and the $4 \pi r^{2} T_{00}^{*}(r) / M_{N}^{*}$ in the right panel, as functions of radial distance $r$. The solid and dashed curves depict the densities respectively from Model I and Model II in free space. The dotted and dot-dashed ones represent respectively those from Model I and Model II in nuclear matter.

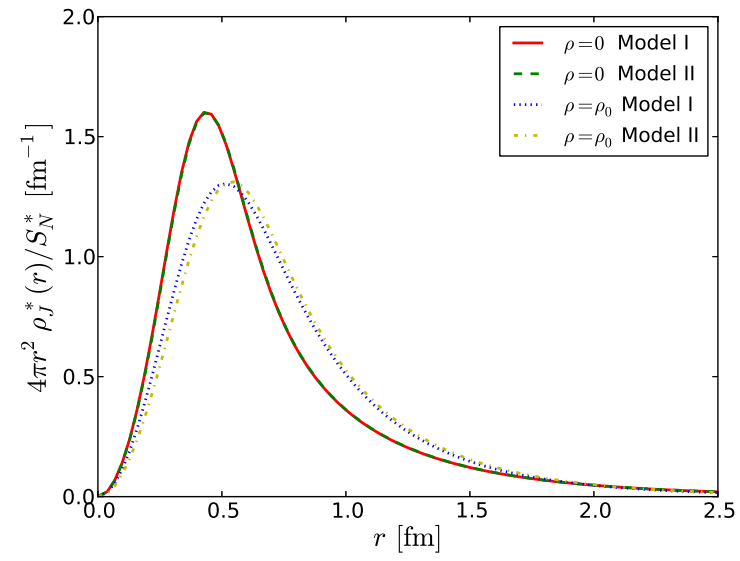

FIG. 2: The angular momentum density $\rho_{J}^{*}(r)$ normalized by the nucleon spin $S_{N}^{*}=1 / 2$ as a function of radial distance $r$. The solid and dashed curves depict the densities respectively from Model I and Model II in free space. The dotted and dot-dashed ones represent respectively those from Model I and Model II in nuclear matter.

changes. ${ }^{3}$ The medium-modified Skyrme model exhibits a similar tendency [20], though the relative increase of $\left\langle r_{J}^{2}\right\rangle^{*}$ is more substantial in that model, see Table II.

Figure 3 depicts the pressure densities in a free nucleon and in a nucleon in nuclear matter. In fact, the pressure density is the most interesting quantity in understanding the modification of the nucleon in nuclear matter, because it reveals vividly the internal dynamics

\footnotetext{
3 The situation becomes different when one considers the effects of explicit isospin symmetry breaking [30] due to the explicit medium modification of the time-dependent part of the $\mathcal{L}_{\pi}^{*}$.
}

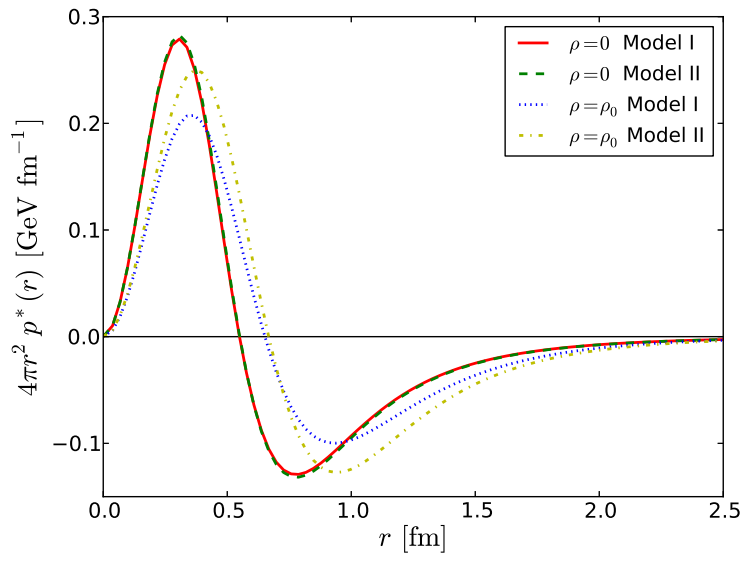

FIG. 3: The pressure density $p^{*}(r)$ as a function of radial distance $r$. The solid and dashed curves depict the densities respectively from Model I and Model II in free space. The dotted and dot-dashed ones represent respectively those from Model I and Model II in nuclear matter.

in the nucleon. As shown in Fig. 3, the pressure density is positive in the inner region, and negative in the outer region. This must be so on general grounds. Positive pressure in the inner region signals repulsive forces, negative pressure in the outer region means attractive forces. Repulsive forces in the inner and attractive forces in the outer parts must balance each other exactly according to Eq. (39).

As in the case of the energy and spin densities, we observe that the pressure density is also broadened in nuclear matter and its modulus is reduced. In Table II, we list the values of the pressure density at $r=0$, i.e. $p^{*}(0)$, and those of $r_{0}^{*}$, which designate the position in which the sign of the pressure density changes. The simulta- 


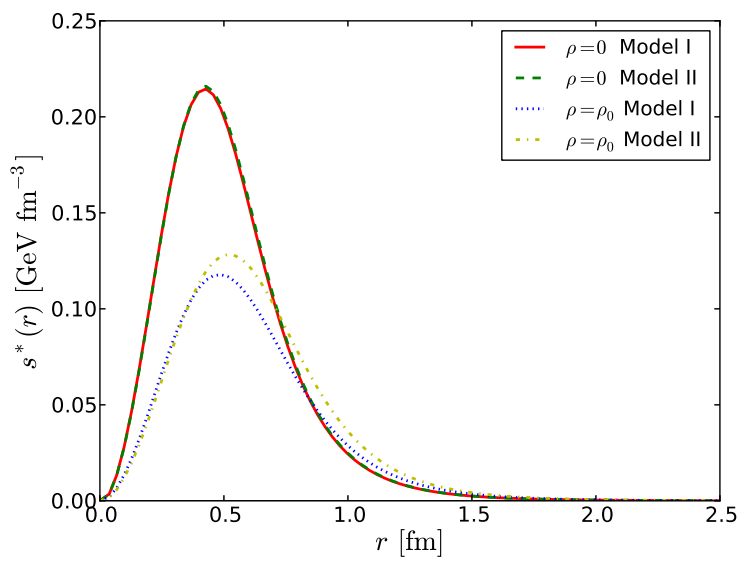

FIG. 4: The shear-force density $s^{*}(r)$ as a function of radial distance $r$. The solid and dashed curves depict the densities respectively from Model I and Model II in free space. The dotted and dot-dashed ones represent respectively those from Model I and Model II in nuclear matter.

neous overall decrease of the modulus of $p(r)$, and the broadening of the pressure density in nuclear matter occur in such a way that the results of the pressure density in medium also comply with the stability condition (39).

It is interesting to compare the pressure distributions in Model I and Model II. One can see that the absolute value of the pressure in Model II is larger in comparison with that in Model I. In the present approach, the nucleon in nuclear environment is a stable object by itself. No "external forces" due to the nuclear medium are required to stabilize it. However, nuclear matter has an impact on the nucleon. In this sense, it is a physically appealing observation that the stiffer nuclear matter and the higher incompressibility in Model II 21] cause the nucleon to be subject to stronger internal forces, for which the magnitude of $p(r)$ is a measure. The stronger repelling inner forces in Model II make moreover the nucleon swell more strongly in nuclear matter. We have seen above the consequence of this: the relative decrease in energy density in the nucleon core is more pronounced in Model II. (See also the second column of the Table凹)

In this context it is interesting to remark that in nuclear matter the mass of the $\omega$-meson is reduced in Model I but not in Model II. Thus, the nucleon is made of heavier degrees of freedom in Model II. Heavier degrees of freedom cause stronger internal forces [16], i.e. a larger magnitude of the pressure distribution which we observe in Fig. 3, (In principle, heavier degrees of freedom also contribute to a larger energy density in the nucleon 16 . However, in this work the parameters are fixed to reproduce the same nucleon mass in free space, and the same mass reduction for a nucleon bound in nuclear matter. Hence this effect on the energy density is not apparent.)

In Fig. 4, we draw the numerical results of the density of shear forces. The conservation of the EMT implies (in a static situation which we encounter here) that the spa- tial components of the EMT satisfy $\partial_{i} T_{i j}=0$. Starting from Eq. (30), one then obtains the differential equation

$$
\frac{2}{3} \frac{\partial s^{*}(r)}{\partial r}+2 \frac{s^{*}(r)}{r}+\frac{\partial p^{*}(r)}{\partial r}=0 .
$$

It is straightforward to verify Eq. (41) by using the expressions (32, 33) for $s^{*}(r)$ and $p^{*}(r)$ and the differential equations (16, 18) for the profile functions $F(r), G(r)$ and $\omega(r)$. Eq. (41) shows that $s^{*}(r)$ and $p^{*}(r)$ are related to each other. However, the shear forces offer a new perspective and therefore equally instructive insights on the internal structure. In order to discuss what we learn from $s^{*}(r)$, let us review the liquid drop analogy. In a liquid drop $s^{*}(r)$ would be given by a delta-function concentrated around the edge of the drop, and the coefficient of the delta-function would be the surface tension. The liquid drop model was explored in [7] to compute the $D$ terms of nuclei. Figure 4 shows that the nucleon is much more diffuse than nuclei: the "delta-function" is strongly smeared out. Nevertheless, the maximum of the shearforce density indicates the "edge of the nucleon" [15]. The effect of the nuclear medium is to shift the peak of $s^{*}(r)$ towards larger $r$ and broaden the shear force distribution. So the nucleon swells and becomes even more diffuse in nuclear medium, which confirms independently what we observed from other densities. Notice that the peak of $s^{*}(r)$ is larger in Model II than in Model I, as the higher "surface tension" has to oppose stronger inner forces in Model II. This reflects independently the stronger response of the nucleon to the stiffer nuclear environment in Model II.

In order to examine the modification of the nucleon in detail, it is instructive to consider the contribution of each meson to the pressure density. In Fig. 5 we plot each contribution of the pure pionic part $\mathcal{L}_{\pi}^{*}$, the pure vector meson part $\mathcal{L}_{\text {kin }}^{*}$ and the interaction part $\mathcal{L}_{V}^{*}$ to the pressure density, which discloses the most prominent feature of the pressure density. As in free space, the pion furnishes a strong attraction with the long-range asymptotics, which reflects the fact that the soliton can never be stabilized by the pion only. Both the vector mesons come into play to make the soliton stable. On the other hand, the Skyrme quartic term stabilizes the soliton in the Skyrme model. Thus, in the present model, the $\rho$ and $\omega$ mesons do play the same role as the Skyrme quartic term [33 35]. The present results for the pressure distribution illustrate this well-known fact.

The roles of the pion and the vector mesons were already discussed in free space [18]. Compared with the results in free space (indicated by the thin lines in Fig. [5), in the nuclear medium the effect of the pion turns out to be noticeably suppressed, while the vector meson kinematic contribution is amplified, and the part due to the interaction term in turn is significantly suppressed. All contributions suffer a shift towards larger $r$, which illustrates the microscopic dynamics responsible for the swelling of the nucleon size in medium.

It is instructive to examine how the interaction part 

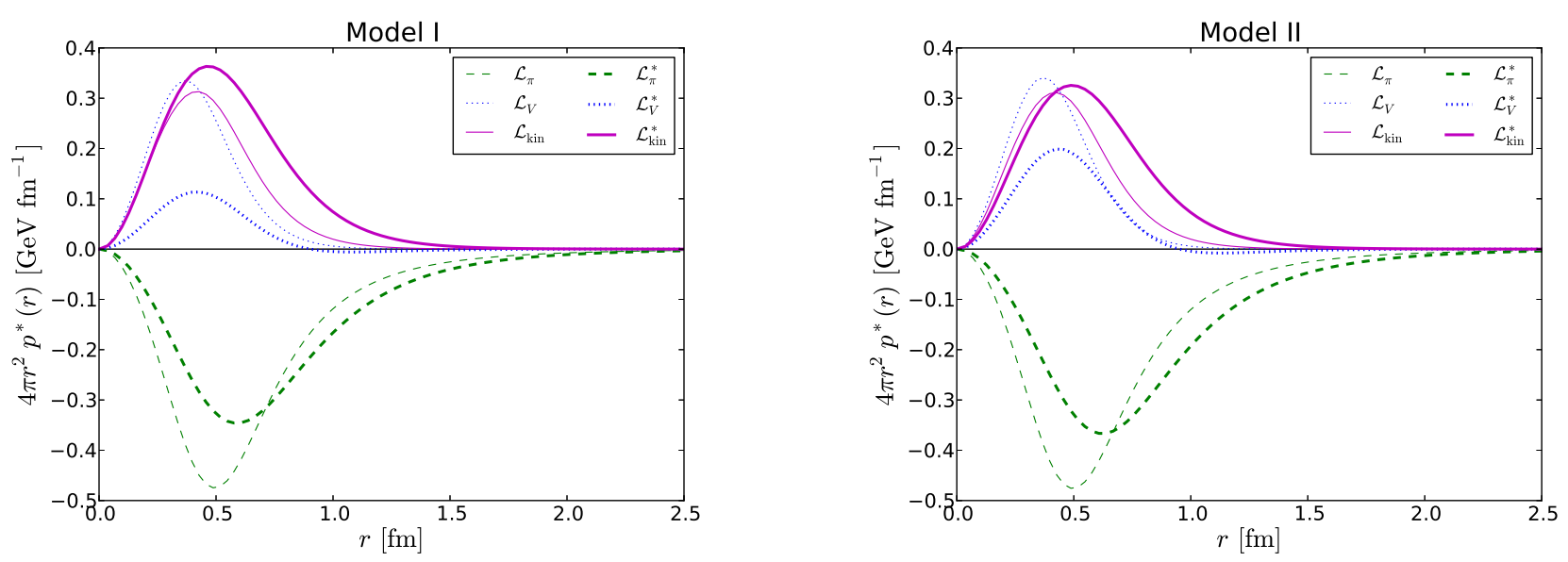

FIG. 5: The contributions of each meson and the kinetic terms to $4 \pi r^{2} p^{*}(r)$. The left panel depicts the results from Model I and the right panel does those from Model II. The dashed curve designates the pion contribution, the dotted one that of the vector mesons, and the solid one that of the kinetic terms. In both panels, the thin curves indicate the results in free space, while thick ones designate those at normal nuclear matter density $\rho_{0}$. The total results for $4 \pi r^{2} p^{*}(r)$ were shown in Fig. 3

$\mathcal{L}_{V}^{*}$ is modified in nuclear medium. The contribution of $\mathcal{L}_{V}^{*}$ in nuclear matter is reduced and turns even negative before $r$ reaches $1 \mathrm{fm}$, see Fig. [5. This change is less pronounced in Model II than in Model I, which reflects the fact that the $\omega$ meson mass is not modified in nuclear medium in Model II. Therefore in Model II the core part of the in-medium nucleon is less modified compared to the free space case, see Fig. 5 .

Another consequence of keeping the omega parameters at their values in free space, is that the positive part of the pressure density from $\mathcal{L}_{\text {kin }}^{*}+\mathcal{L}_{V}^{*}$ turns out to be larger in Model II than in Model I. This implies relatively larger repelling forces in the inner region, and causes a larger swelling of the nucleon in Model II as compared to Model I. (Compare the changes of all mean-squared radii in Model I with those in Model II as listed in Table [I])

With the densities discussed above, we can immediately derive the corresponding EMTFFs. In Figs. 6, 8 we depict the results of the three EMTFFs defined in Eq. (11). Note that the $M_{2}^{*}(t)$ and $J^{*}(t)$ are constrained to be 1 and $1 / 2$ at $t=0$ respectively as shown in Eqs. (37) and (38). Similarly to the in-medium Skyrme model [20], the mass form factor $M_{2}^{*}(t)$ and the spin form factor $J^{*}(t)$ in nuclear medium fall off more rapidly than those in free space. This reflects that the corresponding mean-squared radii in nuclear matter become larger, as we have shown already in Table

The last form factor, which is called the $D$-term form factor, is distinguished from the other two form factors. It is not constrained by any condition. However, the negative sign of $d_{1}$ at $t=0$, which is called the $D$ term, arises as a consequence of stability. The $D$ term is defined in terms of the pressure density

$$
d_{1}^{*}=5 \pi M_{N}^{*} \int_{0}^{\infty} d r r^{4} p^{*}(r)
$$

Recall that the analog integral over $r^{2} p^{*}(r)$ vanishes due to the stability condition (39). This implies that $d_{1}^{*}$ must have a negative value, because $r^{4}$ in the integrand of Eq. (42) lessens the contribution from the inner (positive) part of the pressure density but amplifies that from the outer (negative) part. The explicit value of $d_{1}$ and its change in nuclear matter are given in Tables Iand II. The magnitude of $d_{1}^{*}$ in nuclear matter is larger than that in free space. This can be understood from Fig. 3 in which the pressure density in nuclear matter was shown to be shifted to the outer region and became more strongly negative as $r$ increases. As shown in Fig. 8 , the $d_{1}^{*}$ form factor in nuclear matter falls off more rapidly than that in free space, as the other two form factors do.

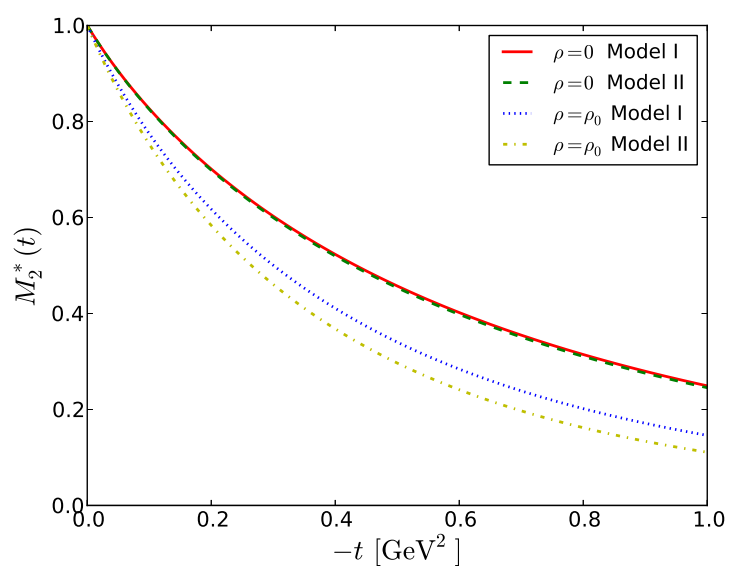

FIG. 6: $M_{2}^{*}(t)$ as a function of $t$. The solid and dashed curves depict the form factor respectively from Model I and Model II in free space. The dotted and dot-dashed ones represent respectively those from Model I and Model II in nuclear matter. 
We note that the comparison of the results from Models I and II explicitly shows that $d_{1}^{*}$ form factor is very sensitive to the stabilization mechanism, i.e. to the interplay between the internal and external forces which stabilize the soliton. This is seen from Table II where the change of $d_{1}^{*}$ in Model II is much larger than in Model I.

Finally, we discuss the mean-squared radius of the EMT trace operator [15]. The form factor of the EMT trace operator can be expressed in terms of the other EMTFFs, so that the mean-squared radius $\left\langle r_{F}^{2}\right\rangle$ (in the notation of Ref. [15]) can be given as

$$
\left\langle r_{F}^{2}\right\rangle^{*}=\left\langle r_{00}^{2}\right\rangle^{*}-\frac{12 d_{1}^{*}}{5 M_{N}^{* 2}}
$$

The importance of this quantity lies in the fact that in QCD in the chiral limit the trace anomaly [36] relates it to the mean-squared radius of the gluonic operator $G^{\mu \nu} G_{\mu \nu}$. Although explicit gluonic degrees of freedom are absent, chiral soliton models allow one to evaluate consistently the trace of the EMT, and thus to obtain in this way insights on this quantity [15]. In Table [ we see that in Model I and Model II $\left\langle r_{F}^{2}\right\rangle=1 \mathrm{fm}^{2}$ in free space. A similar value was obtained in the Skyrme model of Ref. [20] (though it was not explicitly reported there). In the chiral quark-soliton model [15, 16] and a different Skyrme model study 17, comparable values were obtained for $\left\langle r_{F}^{2}\right\rangle$. It is an important observation that the $\pi$ - $\rho-\omega$ model confirms the results from other solitonic approaches concerning the magnitude of $\left\langle r_{F}^{2}\right\rangle$. Thus, the large value of the $\left\langle r_{F}^{2}\right\rangle$ appears to be a robust prediction of chiral soliton models. Their prediction for the mean-squared radius of the trace of the EMT operator is remarkable [15], especially if one confronts it with the QCD sum rule study in which the mean-squared radius of the traceless part of the gluonic contribution to the EMT

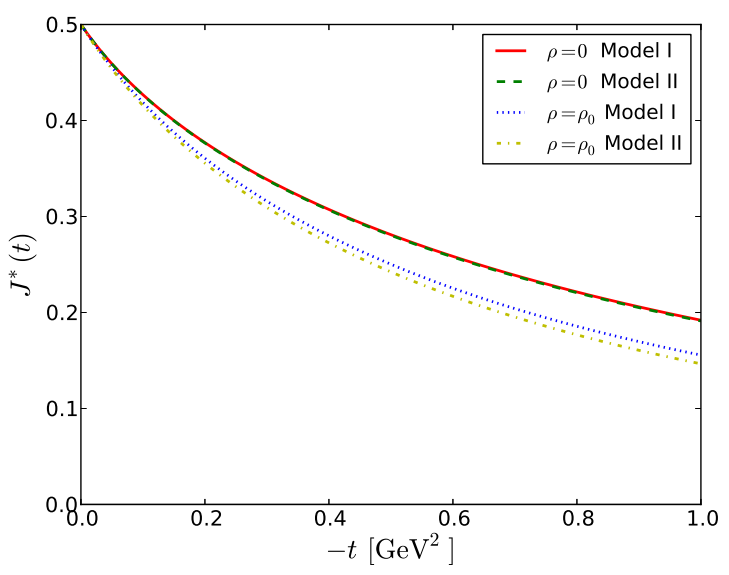

FIG. 7: $\quad J^{*}(t)$ as a function of $t$. The solid and dashed curves depict the form factor respectively from Model I and Model II in free space. The dotted and dot-dashed ones represent respectively those from Model I and Model II in nuclear matter.

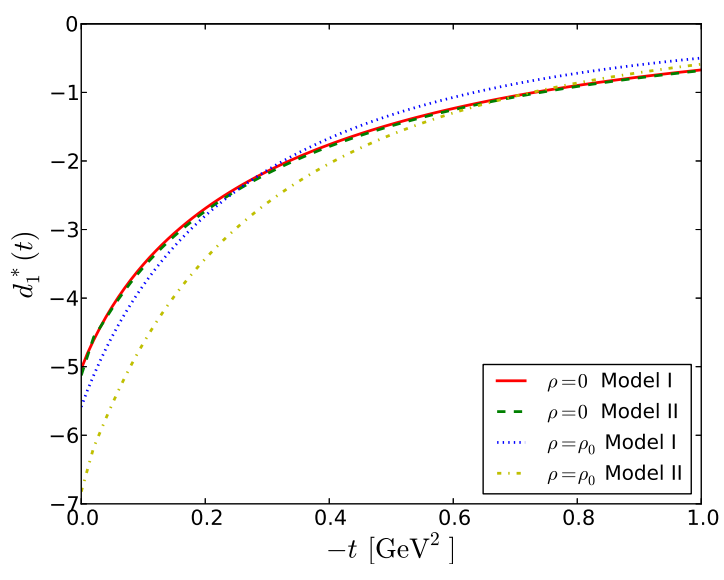

FIG. 8: $d_{1}^{*}(t)$ as a function of $t$. The solid and dashed curves depict the form factor respectively from Model I and Model II in free space. The dotted and dot-dashed ones represent respectively those from Model I and Model II in nuclear matter.

was predicted to be one-order-of-magnitude smaller [37]. ${ }^{4}$

In our context, it is also interesting to explore the effects of the nuclear environment on the trace of the EMT operator. Table II shows that nuclear medium effects increase the mean-squared radius of the EMT trace operator by $30-40 \%$ in Model I and II in the $\pi-\rho-\omega$-soliton framework, and similarly in the Skyrme model [17]. This is rather remarkable. At this point one has to be cautious, because one should take the chiral limit in order to isolate a gluonic contribution to the EMT trace operator. This will be an interesting task for future studies. Here we content ourselves to remark that, by exploring the trace anomaly, studies of the EMT provide a unique opportunity to learn about nuclear medium effects on certain gluonic quantities.

\section{CONCLUSIONS AND SUMMARY}

The present work aimed at investigating the energymomentum tensor form factors in nuclear matter within the framework of the medium-modified $\pi-\rho-\omega$ soliton model. The parameters in the model have been fixed by using the experimental data. The medium functionals, which are introduced to describe the influence of a nuclear environment on the properties of the single soliton, have been fixed by using the analysis of pionic atoms and

\footnotetext{
4 The instanton vacuum approach provides a natural explanation for this observation. The trace of the EMT operator receives one-instanton contributions. In contrast, the traceless part arises from instanton anti-instanton configurations [15] and is therefore of higher order in the instanton packing fraction, which is the small parameter in the instanton vacuum approach describing the diluteness of the instanton medium [38].
} 
the data on low-energy pion-nucleus scattering. We set up two different models, Model I and Model II, by making different assumptions on the KSRF relation. Both provided comparably satisfactory descriptions of medium effects, where Model II describes a stiffer nuclear medium.

We computed the densities associated with the energy momentum tensor, and found that they all exhibited qualitatively similar patterns in nuclear medium: they are diminished in the inner region of the nucleon and broadened towards larger distances. This nicely illustrates the well-known facts that the nucleon swells and its mass is reduced in nuclear matter. In particular, the discussion of the pressure distribution has shown in great detail how the nucleon is stabilized in nuclear medium by the interplay of the different degrees of freedom within the $\pi-\rho-\omega$ soliton model. While the description of the energy-momentum tensor densities is qualitatively similar in Model I and Model II, we made the interesting observation that a stiffer nuclear medium causes the nucleon to experience stronger internal forces. We also derived the three energy-momentum tensor form factors $M_{2}(t)$, $J(t), d_{1}(t)$, and showed that they fall off faster than those in free space, which reflects that in general the meansquared radii become larger in nuclear medium.

In all theoretical studies so far, the $D$-term $d_{1} \equiv d_{1}(0)$ was found to be negative, for the nucleon [12 18], the pion [9], nuclei [7, 22], photons [39], and $Q$-balls [40]. The negative sign of the $D$-term was confirmed also for a nucleon bound in nuclear medium in Ref. [20] and in the present work. Medium effects do not change the generic pattern how internal forces balance to form a stable nucleon. However, they alter the strengths and ranges of the various contributions from the $\pi$-, $\rho$ - and $\omega$-meson degrees of freedom. The $D$-term is the quantity most sensitive to modifications in the interplay of attractive and repulsive internal forces inside the nucleon. The results obtained in this work and in the medium-modified
Skyrme model [20] will shed light on nuclear medium effects on the energy-momentum tensor form factors.

To summarize, the study of the energy-momentum tensor form factors reveals directly the internal structure of the nucleon in both free space and in nuclear matter, of which the latter was in the focus of the present work. It is of great importance to investigate the nucleon inside the isospin asymmetric matter [30], in neutron stars as well as inside real nuclei, where surface effects become essential. Related works are under way.

\section{Acknowledgments}

H.-Ch. $\mathrm{K}$ is grateful to Kyungseon Joo for his hospitality during his visit to University of Connecticut and P. Navratil and R. Woloshyn for their hospitality during his visit to TRIUMF, where parts of the work have been done. This work is supported by the Basic Science Research Program through the National Research Foundation (NRF) of Korea funded by the Korean government (Ministry of Education, Science and Technology), Grant No. 2012-0008469 (J.H.J. and U.Y.) and Grant No. 2012004024 (H.Ch.K.). The work was partly supported by DOE contract DE-AC05-06OR23177, under which Jefferson Science Associates, LLC, operates the Jefferson Lab.

\section{Appendix A: Proof of von Laue condition}

In this Appendix we present an alternative proof of the von Laue condition in Eq. (39). We substitute $r \rightarrow r^{\prime}=$ $\lambda r$ in Eq. (15) (and drop the prime on the integration variable $r^{\prime}$ for simplicity). This yields

$$
\begin{aligned}
M_{\mathrm{sol}}^{*}(\lambda) & =\lambda 4 \pi \int_{0}^{\infty} \mathrm{d} r r^{2}\left\{\frac{1}{g_{\rho}^{2} \zeta_{\rho} r^{2}}\left(G^{2}+\frac{G^{2}(G+2)^{2}}{2 r^{2}}\right)\right\} \\
& +\lambda^{0} 4 \pi \int_{0}^{\infty} \mathrm{d} r r^{2}\left\{\left(\frac{3}{2} g_{\omega} \sqrt{\zeta_{\omega}}\right) \frac{1}{2 \pi^{2} r^{2}} \omega \sin ^{2} F F^{\prime}\right\} \\
& +\frac{1}{\lambda} 4 \pi \int_{0}^{\infty} \mathrm{d} r r^{2}\left\{\alpha_{p} f_{\pi}^{2}\left(\frac{\sin ^{2} F}{r^{2}}+\frac{F^{\prime 2}}{2}\right)+\frac{2 f_{\pi}^{2}}{r^{2}}(1-\cos F+G)^{2}-\frac{1}{2} \omega^{\prime 2}\right\} \\
& +\frac{1}{\lambda^{3}} 4 \pi \int_{0}^{\infty} \mathrm{d} r r^{2}\left\{\alpha_{s} f_{\pi}^{2} m_{\pi}^{2}(1-\cos F)-\zeta_{\omega} g_{\omega}^{2} f_{\pi}^{2} \omega^{2}\right\} .
\end{aligned}
$$

The transformation $r \rightarrow \lambda r$ corresponds to a dilatational variation. The soliton solution is a minimum of the energy functional for any type of variations. Therefore, $M_{\text {sol }}^{*}(\lambda)$ must have a minimum at $\lambda=1$, which implies that

$$
\begin{aligned}
\left.\frac{\mathrm{d} M_{\mathrm{sol}}^{*}(\lambda)}{\mathrm{d} \lambda}\right|_{\lambda=1} & =4 \pi \int_{0}^{\infty} \mathrm{d} r r^{2}\left\{\frac{1}{g_{\rho}^{2} \zeta_{\rho} r^{2}}\left(G^{\prime 2}+\frac{G^{2}(G+2)^{2}}{2 r^{2}}\right)\right\} \\
& -4 \pi \int_{0}^{\infty} \mathrm{d} r r^{2}\left\{\alpha_{p} f_{\pi}^{2}\left(\frac{\sin ^{2} F}{r^{2}}+\frac{F^{\prime 2}}{2}\right)+\frac{2 f_{\pi}^{2}}{r^{2}}(1-\cos F+G)^{2}-\frac{1}{2} \omega^{\prime 2}\right\}
\end{aligned}
$$




$$
\begin{aligned}
& -3 \times 4 \pi \int_{0}^{\infty} \mathrm{d} r r^{2}\left\{\alpha_{s} f_{\pi}^{2} m_{\pi}^{2}(1-\cos F)-\zeta_{\omega} g_{\omega}^{2} f_{\pi}^{2} \omega^{2}\right\} \\
& =3 \times 4 \pi \int_{0}^{\infty} \mathrm{d} r r^{2} p^{*}(r) \\
& \stackrel{!}{=} 0 .
\end{aligned}
$$

In the last step, we explored the fact that the integrand in the energy functional is the same as the expression for the pressure density in Eq. (33) with factor 3 multiplied. This proves the von Laue condition in Eq. (39).

The dilatational variation is a standard procedure to prove the existence of an energy extremum. Equation (A2) implies a relation among the different contributions to the soliton energy, which is sometimes known as a "virial theorem". Analog proofs of the von Laue condition were formulated in the chiral quark-soliton model
15] and Skyrme model [17].

Notice that the Wess-Zumino term does not contribute to $p^{*}(r)$ because the omega-field $\omega(r) \delta_{\mu 0}$ has no spatial components in the Ansatz (14) and hence makes no contribution to the stress tensor. In our proof, we see that the Wess-Zumino term drops out from the von Laue condition (39) since its contribution to the soliton energy scales as $\lambda^{0}$ in $M_{\text {sol }}^{*}(\lambda)$ and therefore does not appear in the dilatational variation in Eq. A2).
[1] H. Pagels, Phys. Rev. 144, 1250 (1966).

[2] D. Müller, D. Robaschik, B. Geyer, F. M. Dittes and J. Hor̆ejši, Fortsch. Phys. 42, 101 (1994).

[3] X. D. Ji, Phys. Rev. D 55, 7114 (1997).

[4] J. C. Collins, L. Frankfurt and M. Strikman, Phys. Rev. D 56, 2982 (1997).

[5] A. V. Radyushkin, Phys. Rev. D 56, 5524 (1997).

[6] X. D. Ji, Phys. Rev. Lett. 78, 610 (1997).

[7] M. V. Polyakov, Phys. Lett. B 555, 57 (2003).

[8] M. V. Polyakov and A. G. Shuvaev, hep-ph/0207153

[9] M. V. Polyakov and C. Weiss, Phys. Rev. D 60, 114017 (1999).

[10] N. Kivel, M. V. Polyakov and M. Vanderhaeghen, Phys. Rev. D 63, 114014 (2001).

[11] K. Goeke, M. V. Polyakov and M. Vanderhaeghen, Prog. Part. Nucl. Phys. 47, 401 (2001).

[12] X.-D. Ji, W. Melnitchouk and X. Song, Phys. Rev. D 56, 5511 (1997).

[13] J. Ossmann, M. V. Polyakov, P. Schweitzer, D. Urbano and K. Goeke, Phys. Rev. D 71, 034011 (2005) hep-ph/0411172.

[14] M. Wakamatsu and Y. Nakakoji, Phys. Rev. D 74, 054006 (2006) hep-ph/0605279. M. Wakamatsu, Phys. Lett. B 648, 181 (2007) hep-ph/0701057).

[15] K. Goeke, J. Grabis, J. Ossmann, M. V. Polyakov, P. Schweitzer, A. Silva and D. Urbano, Phys. Rev. D 75, 094021 (2007).

[16] K. Goeke, J. Grabis, J. Ossmann, P. Schweitzer, A. Silva and D. Urbano, Phys. Rev. C 75, 055207 (2007).

[17] C. Cebulla, K. Goeke, J. Ossmann and P. Schweitzer, Nucl. Phys. A 794, 87 (2007).

[18] J. -H. Jung, U. T. Yakhshiev and H. -Ch. Kim, arXiv:1310.8064 [hep-ph], Jour. Phys. G 41, 055107 (2014).

[19] R. Machleidt, K. Holinde and C. Elster, Phys. Rept. 149, 1 (1987).

[20] H. -Ch. Kim, P. Schweitzer and U. T. Yakhshiev, Phys. Lett. B 718, 625 (2012).
[21] J. -H. Jung, U. T. Yakhshiev and H. -Ch. Kim, Phys. Lett. B 723, 442 (2013).

[22] V. Guzey and M. Siddikov, J. Phys. G 32, 251 (2006).

[23] S. Liuti and S. K. Taneja, Phys. Rev. C 72, 032201 (2005).

[24] S. Scopetta, Phys. Rev. C 79, 025207 (2009).

[25] A. Airapetian et al. [HERMES Collaboration], Phys. Rev. C 81, 035202 (2010).

[26] Ulf-G. Meissner and N.Kaiser, Z. Phys. A 325, 267 (1986).

[27] T. Ericson and W. Weise, Pions and Nuclei (Clarendon, Oxford, 1988).

[28] M. Naruki et al. [KEK-PS E325 Collaboration], Phys. Rev. Lett. 96, 092301 (2006).

[29] M. H. Wood et al. [CLAS Collaboration], Phys. Rev. C 78, 015201 (2008).

[30] U. T. Yakhshiev, Phys. Rev. C 88, 034318 (2013).

[31] M. von Laue, Ann. Phys. (Leipzig) 340, 524 (1911).

[32] I. Białynicki-Birula, Phys. Lett. A 182, 346 (1993).

[33] T. Fujiwara, Y. Igarashi, A. Kobayashi, H. Otsu, T. Sato, S. Sawada, Prog. Theor. Phys. 74, 128 (1985).

[34] Y. Igarashi, M. Johmura, A. Kobayashi, H. Otsu, T. Sato, S. Sawada, Nucl. Phys. B 259, 721 (1985).

[35] Ulf-G. Meissner, N. Kaiser, A Wirzba, W. Weise, Phys. Rev. Lett. 57, 1676 (1986).

[36] S. L. Adler, J. C. Collins and A. Duncan, Phys. Rev. D 15, 1712 (1977).

J. C. Collins, A. Duncan and S. D. Joglekar, Phys. Rev. D 16, 438 (1977).

[37] V. M. Braun, P. Gornicki, L. Mankiewicz and A. Schafer, Phys. Lett. B 302, 291 (1993).

[38] D. Diakonov, M. V. Polyakov and C. Weiss, Nucl. Phys. B 461, 539 (1996) hep-ph/9510232.

[39] I. R. Gabdrakhmanov and O. V. Teryaev, Phys. Lett. B 716, 417 (2012).

[40] M. Mai and P. Schweitzer, Phys. Rev. D 86, 076001 (2012); Phys. Rev. D 86, 096002 (2012). 